Methods: Retrospective review of patients seen between July 2018 and July 2019 at Purley Memorial Hospital, covering a large urban borough. Patients included in the study had prevalent moderate and severe VFFs (defined by Ghent criteria). Results: 84 patients were included, of which there were 71 females and 13 males. Median age was 76 (IQR $67-82$ ) and BMI was 25 (IQR $21-27$ ), with $6 \%$ of patients noted to be underweight (BMI $<18.5)$. $38 \%$ of patients had a family history of osteoporosis and $21 \%$ of patients reported a history of parental hip fracture. $21 \%$ of patients had experienced recurrent falls. Over half $(56 \%)$ of patients were noted to have had 2-or-more previous fragility fractures. Of the female cohort, $24 \%$ had early menopause, $13 \%$ were nulliparous and $1 \%$ had late menarche. Of the modifiable risk factors, alcohol (24\%), sedentary lifestyle $(21 \%)$ and smoking (13\%) were the most common. Thyroid-disease $(18 \%)$ was the most common medical condition associated, followed by coeliac/malabsorption (7\%), endocrinopathy (6\%) and COPD (6\%). Proton Pump Inhibitors (PPIs) and steroids were the most common medications associated with $16 \%$ and $14 \%$ of cases respectively. $73 \%$ of patients had good calcium intake and $62 \%$ had calcium supplements. Overall, 27 patients had 3-or-fewer risk factors, 35 patients had 4-to-6 risk factors, 19 had 7-to-9 risk factors, and 3 had greater than 10 risk factors.

Conclusion: Our study identified several risk factors and their prevalence. The majority of patients were female, of which $24 \%$ had early menopause. Several modifiable risk factors such as low BMI, alcohol, smoking and sedentary lifestyle were commonly seen, emphasising the need to identify these risk factors in the initial consultation. Over half of patients $(56 \%)$ had had 2-or-more previous nonVFFs, suggesting the need to screen for VFFs in patients with multiple fragility fractures at baseline assessment. A number of co-existing medical conditions were also observed with thyroid disease being the most prevalent. PPIs and steroids were found to be important risk factors, which may lead to the consideration of alternative medications. Overall, it was found that patients had multiple risk factors, thus it is important to have a holistic assessment of VFF patients and a multi-system management approach to prevent future fractures.

Disclosure of Interests: None declared.

DOI: 10.1136/annrheumdis-2021-eular.2201

\section{AB0617 BISPHOSPHONATES DANS L'OSTÉOPOROSE ET LE RISQUE D'OSTÉONÉCROSE DE LA MÂCHOIRE, ENVIRON 896 CAS}

\section{A. R. Halidou ${ }^{1}$, K. Nassar ${ }^{1}$, S. Janani ${ }^{1} .{ }^{1}$ Université Hassan II, médecine, Casablanca, Morocco}

Background: Bisphosphonates (BF) are used in the treatment of osteoporosis, Paget's disease of bone, hypercalcemia and in patients with cancer. When used to treat osteoporosis, the optimal duration of treatment is 3 to 5 years; however, their long-term use has been rarely associated with osteonecrosis of the jaw. Objectives: To assess the risk of developing osteonecrosis of the jaw in patients followed for osteoporosis and on bisphosphonates (BP).

Methods: Type of study: retrospective study conducted at the rheumatology department of the IBN ROCHD CHU in Casablanca.

Duration: from October 2013 to October 2020 (7 years).

Inclusion criteria: all patients followed for osteoporosis in the weakening osteopathies unit of the bone and treated with oral or intravenous bisphosphonates. Exclusion criteria: patients followed for other than osteoporosis.

Results: 896 patients were treated during this period. The average age was 62.74 years (28 to 90 years), of which $85.16 \%$ were women and $14.84 \%$ were men, for a sex ratio (F/M) of 5.74 . As a history, $18.75 \%$ of patients are diabetic, $26.56 \%$ followed for breast neoplasm, $14.06 \%$ of patients had received long-term corticosteroid therapy for various pathologies such as chronic inflammatory rheumatism. Osteoporosis was postmenopausal in 687 patients, ie $76.67 \%$ of cases, $14.06 \%$ after long-term corticosteroid therapy, $8.15 \%$ following hormone therapy (anti-aromatases) and $6.92 \%$ following chemotherapy; note that $18.16 \%$ of these patients were found in at least two of the situations. The mean bone mineral density (BMD), T-score pair considered in all [T-score (BMD)] is $-3(0.736)$ in the lumbar spine (L1-L4), -2.9 (0.658) at the femoral neck, $-2.6(0.804)$ at the total hip before the start of treatment. $69.97 \%$ of the patients were put on Alendronic acid, $12.50 \%$ on Residronic acid, $10.93 \%$ on Zolidronic acid, $3.46 \%$ on Pamidronic acid and $3.14 \%$ received Strontium Ranelate, note that before the start of the treatment all the patients benefited from a dental consultation followed by care of any lesions, the bisphosphonates were only introduced after having ruled out all their dental contraindications, the average duration of treatment for all the molecules was 4.71 years ( 2 to 5 years) and no patient developed osteonecrosis of the jaw. The change in control BMD on average after 2 years of treatment was $-2.7(0.782)$ at the lumbar spine, -2.6 (0.749) at the femoral neck and -2.4 (0.713) at the hip total, after 5 years $-2.4(0.874)$ at the spine, $-2.1(0.809)$ at the femoral neck and -1.93 (861) at the total hip.

Conclusion: The occurrence of ONJ in the treatment of osteoporosis with the use of BFs is rare, and appears to be unpredictable; but maintaining therapeutic caution, consisting in diagnosing and treating any dental lesions before starting treatment, can considerably reduce or even cancel the risk of occurrence; especially in patients treated with long-term intravenous pamidronate.

\section{REFERENCES:}

[1] Dr Halidou Idrissa Abdoul-Rahamane, Pr Kawtar Nassar, PR Saadia Janani.

[2] Rheumatology department of the IBN ROCHD CHU in CASABLANCA. Cas ablanca Faculty of Medicine and Pharmacy. Hassan II University. Morocco

Disclosure of Interests: None declared.

DOI: 10.1136/annrheumdis-2021-eular.2399

\section{AB0618 GLUCOCORTICOID INDUCED OSTEOPOROSIS PREVENTION IN THE OUTPATIENT RHEUMATOLOGY CLINIC}

Z. Vaghaiwalla ${ }^{1}$, G. Kaeley ${ }^{1}{ }^{1}$ University of Florida College of MedicineJacksonville, Rheumatology, Jacksonville, United States of America

Background: Patients with rheumatic disease are at risk of developing glucocorticoid induced osteoporosis (GIOP) as many are prescribed systemic ora glucocorticoids as an adjunct to their maintenance therapy. Based on the 2017 ACR Guideline for the Prevention and Treatment of Glucocorticoid-Induced Osteoporosis, a good practice recommendation exists that "initial clinical fracture risk assessment should be performed as soon as possible, but at least within six months of the initiation of long term glucocorticoid treatment. " Long-term glucocorticoid use is defined by duration of 3 months or greater. Fracture risk assessment should include dual energy-ray absorptiometry (DEXA) scan. Patients on greater than or equal to $2.5 \mathrm{mg}$ of prednisone should be treated with an optimal dose of calcium and vitamin $D$ and may benefit from oral bisphosphonate as primary prevention against GIOP if their fracture risk is moderate to high. ${ }^{1}$

Objectives: The aim of this Quality Improvement Project is to assess the current status of provider implementation of GIOP recommendations in the rheumatology clinic. Ultimate goal is to improve osteoporosis prevention in the rheumatology clinic.

Methods: We conducted a retrospective chart review of 60 patients in two outpatient rheumatology clinics. Clinic 1 follows patients with lower socioeconomic status and Clinic 2 follows patients with higher socioeconomics. Inclusion criteria were patients on long-term glucocorticoid use, defined as at least 3 months of corticosteroid use of at least $2.5 \mathrm{mg}$ prednisone daily, as well as age less than 65 Females aged 65 or older were omitted to prevent overlap of the United States Preventative Taskforce recommendation for all women $\geq 65$ years to be screened for osteoporosis with DEXA scans. ${ }^{2}$ DEXA scan orders, calcium and vitamin D prescriptions, and osteoporosis medication prescriptions were abstracted. After baseline data obtained, intervention of education of the rheumatology fellows and faculty, and internal medicine residents in the guidelines for GIOP prevention was implemented. In addition, a smartphrase in the electronic medical record was created for provider use when treating patients on chronic corticosteroids. Subsequently, two audit cycles were completed for retrospective chart review.

Results: Upon completion of second audit cycle, there was no change in percentage of DEXA scan orders at Clinic 1, however there was a $10 \%$ overal improvement in DEXA scan orders in the Clinic 2.

In terms of Calcium and Vitamin D prescriptions, there was an overall improvement in both clinics of $19.7 \%$ and $13.3 \%$ in Clinics 1 and 2 respectfully after the second audit cycle.

Additionally, there was a $3.4 \%$ increase in osteoporosis medication prescriptions overall subsequent to the second audit cycle in Clinic 1. However in Clinic 2 there was an overall decrease in osteoporosis medication prescriptions of $6.6 \%$.

\begin{tabular}{|c|c|c|c|}
\hline Clinic 1 & Prior to Audit & Audit cycle 1 & $\begin{array}{c}\text { Audit } \\
\text { cycle } 2\end{array}$ \\
\hline Patient percentage without DEXA scan orders & $30 \%$ & $33.30 \%$ & $30 \%$ \\
\hline Patient percentage without Vitamin D/Calcium orders & $26.40 \%$ & $8.30 \%$ & $6.70 \%$ \\
\hline $\begin{array}{l}\text { Patient percentage with osteoporosis medication } \\
\text { orders }\end{array}$ & $23.30 \%$ & $8.30 \%$ & $26.70 \%$ \\
\hline \multicolumn{4}{|l|}{ Clinic 2} \\
\hline Patient percentage without DEXA scan orders & $50 \%$ & $37.00 \%$ & $40 \%$ \\
\hline Patient percentage without Vitamin D/Calcium orders & $30 \%$ & $26.00 \%$ & $16.70 \%$ \\
\hline $\begin{array}{l}\text { Patient percentage with osteoporosis medication } \\
\text { orders }\end{array}$ & $23.30 \%$ & $11.10 \%$ & $16.70 \%$ \\
\hline
\end{tabular}

Conclusion: Overall, the results of the intervention were strongest for improvements in Vitamin D and Calcium orders in both clinics. Improvements in DEXA scan orders and osteoporosis medications were present in Clinic 2 and not present in Clinic 1. This reveals continued efforts and education of providers need to be made for improvement in bone health monitoring.

\section{REFERENCES:}

[1] Buckley, Lenore, et al. "2017 American College of Rheumatology Guideline for the Prevention and Treatment of Glucocorticoid-Induced 
Osteoporosis." Arthritis \& Rheumatology, vol. 69, no. 8, June 2017, pp. 15211537., doi:10.1002/art.40137.

[2] Final Recommendation Statement: Osteoporosis to Prevent Fractures: Screening. U.S. Preventive Services Task Force. July 2019.

Disclosure of Interests: None declared.

DOI: 10.1136/annrheumdis-2021-eular.2583

\section{AB0619 CLINICAL RISK FACTORS, BONE DENSITY AND FALL'S HISTORY AND FRACTURES RELATION: STUDY BY HOSPITAL SURVEY ABOUT 448 CASES}

K. Nassar ${ }^{1}$, S. Janani ${ }^{2} .{ }^{1}$ Ibn Rochd University Hospital, Hassan II University of Medicine and Pharmacy, Rheumatology Department, CASABLANCA, Morocco; ${ }^{2}$ Ibn Rochd University Hospital.. Hassan II University of Medicine and Pharmacy, Rheumatology Department, CASABLANCA, Morocco

Background: Osteoporosis is a disease that affects bone mineral density (BMD) and bone microarchitecture at the origin of an increased risk of fracture. The reduction in bone density assessed by dual-energy $X$ ray absorptiometry (DXA) and fall history represent the first two risk factors of non-vertebral fracture after menopause. Given the high prevalence of osteoporosis among fallers subjects with common risk factors, causing a surisk of fracture. Several publications including the osteoporosis recommendations indicate DXA in women experienced falls in search of bone fragility may justify a treatment for osteoporosis, especially as the FRAX tool does not include at present the fall of history, an important parameter in the assessment of fracture risk.

Objectives: Because of the low attention given to the evaluation of falls'risk, the main objective of the study was to determine the prevalence and the relationship between the past history of fall, reduction of bone mineral density and prevalent fracture.

Methods: Transversal and descriptive epidemiological cohort study conducted for 24 months in 448 patients referred by physicians regularly use prescription of BMD. The realization of this exploration by the same DXA-Hologic in the rheumatology department at Ibn Rochd University Hospital was the criterion for entry into the study. All patients were interviewed on the same day on the risk factors for osteoporosis and fractures justifying the prescription of a BMD.

Results: Data included 413 women and 35 men. Most women were postmenopausal $(89.6 \%)$. The average age was 59 years $(\sigma=13.40)$, $33.7 \%$ were $\geq 65$ years. The mean BMI was at $27.50(\sigma=5,2) .18 .5 \%$ of patients had at least a history of falls in the previous 12 months and $22.5 \%$ a history of fracture after a low-energy trauma. $42.9 \%$ were osteoporotic and $57.1 \%$ had osteopenia in at least one of these sites: lumbar spine, femoral neck, total hip. We did not find significant association between BMD osteoporosis and fall history $(p=0.916)$. Thus, cases of osteoporosis fractures were not statistically associated with a fall $(p=0.170)$. Also, the falls were occurring than $18.4 \%$ of osteopenic patients $(p=0.220)$. However, in our study, the fall was an independent risk factor for fracture $(p=0.003)$ and osteopenic fractures were significantly higher among fallers $(p=0.009$ and 0.006 respectively, a drop of history and at least one past fall history).

Conclusion: The fall history is a independent risk factor for fracture. This risk is particularly important in case of fragility bone densitometry. The clinical history and fall risk factors should be taken into consideration in the assessment of fracture risk and in the anti-ostoporotique treatment strategy. Thus, the fall seems to be a legitimate indication for DXA and as a parameter to be integrated into the assessment of fracture risk by the FRAX score.

REFERENCES:

[1] MH. Edwards, K. Jameson, H. Denison, NC. Harvey, Sayer A. Aihie, EM Dennison, and C Cooper. Clinical risk factors, bone density and fall history in the predection of incident fracture among men and women. Bone. 2013; 52(2): 541-547.

Table 1. Multivariate Logistic Regression Results: Adjustment for predictors factors of falls

\begin{tabular}{lcc}
\hline Variables & OR (95\% IC) & p-value \\
\hline Age $\geq 65$ ans & $0,986(0,412-2,357)$ & 0,974 \\
Past history of fracture & $4,271(1,719-10,611)$ & 0,02 \\
Walking aid & $11,214(2,815-44,670)$ & 0,01 \\
Vision disturbances & $8,587(3,540-20,829)$ & $<0,001$ \\
Rhumatoid arthritis & $8,047(2,218-29,192)$ & 0,02 \\
Diabete & $3,194(1,217-8,382)$ & 0,018 \\
Corticosteroid $\geq 3$ mois & $0,603(0,156-2,331)$ & 0,463 \\
Smoking & $0,241(0,013-4,518)$ & 0,341 \\
Ostéopenia $(-1 \leq$ T-score $<-2,5)$ & $2,204(0,875-5,552)$ & 0,094 \\
\hline
\end{tabular}

Disclosure of Interests: None declared.

DOI: 10.1136/annrheumdis-2021-eular.2705

\begin{tabular}{l|l}
\hline AB0620 & MODIFICATION OF THE DECISION OF THE ANTI- \\
OSTEOPOROSIS TREATMENT AFTER PERFORMING \\
THE VFA
\end{tabular}

H. Tantaoui ${ }^{1}$, K. Nassar ${ }^{1}$, S. Janani ${ }^{1} .{ }^{1}$ Faculty of Medicine and Pharmacy, Hassan II University, Ibn Rochd University Hospital Center, Rheumatology, Casablanca, Morocco

Background: Spinal fractures are the most common of all osteoporotic fractures. Its diagnosis is essential, because the discovery of a vertebral fracture testifies the gravity of osteoporosis and modifies the therapeutic intervention threshold by justifying a specific anti-osteoporosis treatment. The evolution of densitometers now makes it possible to take a true X-ray image using software called "Vertebral Fracture Assessment" or VFA

Objectives: To assess the impact of VFA results on therapeutic decision-making after measuring bone mineral density.

Methods: We conducted a retrospective and descriptive study in the rheumatology department.

We included all patient followed at the consultation for bone pathologies, in whom a measurement of bone mineral density and a supplementation of VFA were performed. Clinical data, BMD, VFA and the therapeutic decision by anti-osteoporosis treatment before and after VFA were collected.

Results: Sixty-one patients were included. The mean age was 62.8 years [38 - 85 years]. Sex ratio (female / male) was 19.3. At BMD level, patients with osteoporosis and osteopenia were $49 \%$ and $51 \%$ respectively. VFA objectified at leas one spinal fracture in $64 \%$ of patients. Prior to VFA, anti-osteoporosis therapy was indicated to $49 \%$ of patients, based on clinical data and BMD. After performing VFA, the prescription of anti-osteoporosis therapy was indicated to $80 \%$ of patients.

Conclusion: Patients who had no indication for osteoporosis treatment based on BMD data, VFA was able to modify their therapeutic treatment by detecting vertebral fractures in patients who had back pain.

Disclosure of Interests: None declared.

DOI: 10.1136/annrheumdis-2021-eular.3318

\section{$\mathrm{AB} 0621$ \\ THE MAIN CAUSES OF MORTALITY IN PATIENTS WITH OSTEOPOROTIC HIP FRACTURES}

J. Averkieva ${ }^{1}$, M. Letaeva ${ }^{1}$, O. Malyshenko', M. Koroleva ${ }^{1}$, T. Raskina ${ }^{1}$.

${ }^{1}$ Kemerovo State Medical University, Department of Propedeutics of Internal Medicine, Kemerovo, Russian Federation

Background: Increasing life expectancy is one of the main achievements of modern medicine. However, longevity is inevitably associated with the growth of so-called "age-associated diseases," among which osteoporosis occupies an important place.

Objectives: To study the causes of mortality in patients with hip osteoporotic fractures during 12 months after injury.

Methods: Traced the causes of mortality 432 patients with osteoporotic hip fractures were under observation: 328 women and 104 men. The mean age of women was $75.4 \pm 9.27$ years, the mean age of men was $71.5 \pm 10.39$ years.

Results: This study traced the causes of deaths by the main classes of ICD10. It was found that 6 months after a hip fracture in $63(66.3 \%)$ patients, death occurred from diseases of the circulatory system: in men - 13 (59.0\%) cases and in women - $50(68.4 \%)(p=0.9)$. Respiratory diseases, as a cause of death were identified in $18(18.9 \%)$ patients (in men - $5(22.9 \%)$ cases and in women - $13(17.8 \%)(p=0.1))$, neoplasms - in $10(10.5 \%)$ men and women: $2(9.1 \%)$ and $8(10.9 \%)$ cases, respectively $(p=0.5)$. Diseases of the digestive system accounted for a small percentage of the total number of deaths $(3.2 \%)$, without statistically significant differences by gender (in men - $1(4.5 \%)$ case and in women $-2(2.9 \%)$ cases $(p=0.75))$. Among the dead men, $1(1.1 \%)$ death from an infectious disease was registered. 12 months after the fracture, most of the deaths in both men and women were due to diseases of the circulatory system. The total number of deaths was $93(67.8 \%)$ : in men - 22 (66.0\%) cases, in women - $71(68.3 \%)(p=0.65)$. Respiratory diseases caused death in 23 $(16.8 \%)$ patients: in men $-5(15.1 \%)$ cases and in women - $18(17.3 \%)(p=0.31)$ There is an increase in the number of deaths from neoplasms to $15(10.9 \%)$ cases without statistically significant differences by gender: in men - $3(9.09 \%)$ cases and in women - $12(11.5 \%)(p=0,45)$. Diseases of the digestive system, as a cause of death, were identified in $5(3.6 \%)$ men and women $(2(6.06 \%)$ and $3(2.9 \%)$ cases, respectively $(p=0.1))$. No new cases of deaths from infectious diseases were registered. 